\title{
Association of EPAS1 and PPARA Gene Polymorphisms with High-Altitude Headache in Chinese Han Population
}

\author{
Yang Shen $\mathbb{D}^{1,2}$ Jihang Zhang, ${ }^{1,2}$ Jie Yang, ${ }^{1,2}$ Chuan Liu, ${ }^{1,2}$ Shizhu Bian, ${ }^{1,2}$ Chen Zhang, ${ }^{1,2}$ \\ Jie Yu, ${ }^{1,2}$ Xubin Gao, ${ }^{1,2}$ and Lan Huang $\mathbb{1}^{1,2}$ \\ ${ }^{1}$ Institute of Cardiovascular Diseases of PLA, Xinqiao Hospital, Army Medical University, Chongqing, China \\ ${ }^{2}$ Department of Cardiology, Xinqiao Hospital, Army Medical University, Chongqing, China \\ Correspondence should be addressed to Lan Huang; huanglan260@126.com
}

Yang Shen and Jihang Zhang contributed equally to this work.

Received 2 September 2019; Revised 9 December 2019; Accepted 14 December 2019; Published 24 February 2020

Academic Editor: Salvatore Gallone

Copyright (c) 2020 Yang Shen et al. This is an open access article distributed under the Creative Commons Attribution License, which permits unrestricted use, distribution, and reproduction in any medium, provided the original work is properly cited.

\begin{abstract}
Background. High-altitude headache (HAH) is the most common complication after high-altitude exposure. Hypoxia-inducible factor- (HIF-) related genes have been confirmed to contribute to high-altitude acclimatization. We aim to investigate a possible association between HIF-related genes and HAH in the Chinese Han population. Methods. In total, 580 healthy Chinese Han volunteers were recruited in Chengdu $(500 \mathrm{~m})$ and carried to Lhasa $(3700 \mathrm{~m})$ by plane in 2 hours. HAH scores and basic physiological parameters were collected within 18-24 hours after the arrival. Thirty-five single nucleotide polymorphisms (SNPs) in HIF-related genes were genotyped, and linkage disequilibrium (LD) was evaluated by Haploview software. The functions of SNPs/haplotypes for HAH were developed by using logistic regression analysis. Results. In comparison with wild types, the rs4953354 " $G$ " allele $(P=0.013)$, rs6756667 "A" allele $(P=0.036)$ in EPAS1, and rs6520015 "C" allele in PPARA $(P=0.009)$ were significantly associated with decreased risk of HAH. The rs7292407-rs6520015 haplotype "C-C" in PPARA was identified as a protective factor $(P=0.030)$. Importantly, EPAS1 and PPARA genes have synergistic effects in decreasing HAH risk $(P=0.003)$. Conclusions. EPAS1 and PPARA polymorphisms were associated with HAH in the Chinese Han population. Our findings pointed out potentially predictive gene markers, provided new insights into understanding pathogenesis, and may further provide prophylaxis and treatment strategies for HAH.
\end{abstract}

\section{Introduction}

High-altitude headache (HAH) is the most frequent complaint in lowlanders who ascend from plain area to high altitude and occurs either as an isolated symptom or as a part of acute mountain sickness (AMS) [1-4]. According to Lake Louise AMS scoring system revised in 2018, headache is an indispensable symptom [5]. HAH is defined by the International Headache Society as a disorder that typically develops within 24 hours after rapidly ascending to high altitude $(\geq 2500 \mathrm{~m})$ and resolves within 8 hours after descending [6]. Although $\mathrm{HAH}$ is not severe altitude sickness, it brings confusion, discomfort, and the poor state of emotional wellbeing and may even progress to life-threatening high-altitude cerebral edema (HACE) or high-altitude pulmonary edema (HAPE) [7]. About $80 \%$ of lowlanders are susceptible to $\mathrm{HAH}$ at altitudes higher than $3000 \mathrm{~m}$; therefore, HAH has become a public health problem that demands prompt solution [4].

Multiple factors contribute to the development of $\mathrm{HAH}$, including a history of headache, young age, female gender, obesity, strenuous exercise, dehydration, reaching altitude, and ascending speed, particularly at a speed of greater than $300-500 \mathrm{~m} /$ day $[5,8,9]$. With the increase of altitude, atmospheric pressure decreases which leads to a reduction of arterial oxygen pressure and activation of chemoreceptors. Basic pathophysiological changes include hypoxaemia, hyperventilation, and consequent cerebrovascular responses in the brain [10-12]. It is of vital importance to deliver sufficient oxygen to the brain via precise regulation of cerebral 
blood flow ( $\mathrm{CBF}$ ) to produce enough adenosine triphosphate and maintain the normal physiological function of brain tissue [13]. The increase of CBF elevates intracranial pressure, leading to brain swelling. Ross RT proposed the "tightfit hypothesis" that people with a greater ratio of cranial cerebrospinal fluid to brain volume could compensate for the displacement of cerebrospinal fluid and be more tolerated to brain swelling and thus be less susceptible to $\mathrm{HAH}$ [14]. Yet another explanation emphasized that hypoxia caused cerebral edema and $\mathrm{HAH}$ by inducing damage of blood-brain barrier or by stimulating neurohumoral and hemodynamic responses, leading to cerebral vasodilation and overperfusion of microvascular beds via the release of inflammatory mediators $[11,15]$. From previous studies of our group, Bian SZ and Guo WY reported that HAH patients featured higher vertebral artery diastolic velocity, higher heart rate (HR), higher self-rating anxiety scale score, and lower arterial oxygen saturation $\left(\mathrm{SaO}_{2}\right)$ according to cohort study [16-18]. However, the exact pathophysiological mechanisms of $\mathrm{HAH}$ are multifactorial and far from specific elucidation.

Lack of oxygen is a common problem that people face at high altitude; thus, hypoxia is considered as the initial factor that triggers the development of HAH and AMS. However, lowlanders are more susceptible to high-altitude illness and are more likely to get brain function impairment than highlanders [19]. The concentration of blood hemoglobin, a typical indicator of hypoxia, is lower in Tibetans than in lowlanders when exposed to the same altitude [20]. Recently, genome-wide association studies have confirmed that genetic background differs between lowlanders and highlanders, which means that genomic loci have undergone natural selection in highlanders for hundreds of generations. Previous studies on the association between hypoxia adaptation and gene polymorphisms have focused on hypoxia-inducible factor 1 alpha subunit (HIF-1A, encoding HIF-1 $\alpha$ ), hypoxia-inducible factor 1 alpha subunit inhibitor (HIF1AN, encoding HIF-1 $\alpha$ inhibitor), egl-9 family hypoxia-inducible factor 1 (EGLN1, encoding prolyl hydroxylase domain-containing proteins-2, PHD2), endothelial PAS domain protein 1 (EPAS1, encoding HIF-2 $\alpha$ ), and peroxisome proliferator-activated receptor alpha (PPARA, encoding peroxisome proliferator-activated receptor $\alpha, \operatorname{PPAR} \alpha$ ) [21-27]. Family of HIF transcription factors play key roles in cellular and systemic adaptation including erythropoiesis, iron metabolism, vascular growth, and permeability [24]. Moreover, EPAS1 has been approved to be associated with high-altitude adaptation in Tibetans and the occurrence of high-altitude illness including HAPE, HACE, and high-altitude polycythemia (HAPC) [28-30]. PPAR $\alpha$ is relevant to energy metabolism, specifically the fatty acid beta-oxidation in mitochondrial and peroxisomal under hypoxia conditions. The inhibition of PPAR $\alpha$ function may increase organs' susceptibility to oxidative damage [31, 32]. Furthermore, HIF1A, HIF1AN, and EGLN1 participate in oxidative stress and the occurrence of metabolic diseases through HIF mediated transcriptional regulatory mechanism [21, 33-35]. So, we propose our hypothesis that associations may exist between HIF-related genetic factors and the susceptibility or resistance to HAH in the Chinese Han population.
For the above, the specific molecular mechanism of $\mathrm{HAH}$ remains unclear and the association between genetic variants and $\mathrm{HAH}$ after acute high-altitude exposure has been poorly understood. In the prospective cohort study, 35 SNPs in EPAS1, EGLN1, HIF1A, HIF1AN, and PPARA genes are selected, and the associations between SNPs and HAH in Chinese Han population are evaluated after rapidly ascending to Lhasa $(3700 \mathrm{~m})$ by plane. We aim to enhance the understanding of the concrete mechanisms of $\mathrm{HAH}$, provide useful predictive information, and contribute to developing prevention and treatment strategies of $\mathrm{HAH}$.

\section{Materials and Methods}

2.1. Study Population. The study enrolled 580 unrelated Chinese Han males aged from 18 to 45 years in Chengdu $(500 \mathrm{~m})$ in June 2012. All volunteers were military officers and soldiers, and detailed information including birthplace and permanent residence was collected. Han people who lived in the plain area permanently without high-altitude exposure history within 6 months were included. Subjects enrolled in the study took health examinations in Xinqiao Hospital (Chongqing, China) prior to the trip and would be excluded if they had a history of migraine or nonmigraine headache, cardiovascular diseases, respiratory diseases, neurological diseases, cerebral vascular diseases, cancer, and liver or kidney dysfunction. Individuals taking medicine or receiving prevention measures were also excluded.

2.2. Procedure and Data Collection. According to the study design, 580 participants were carried from Chengdu $(500 \mathrm{~m})$ to Lhasa $(3700 \mathrm{~m})$ by plane in 2 hours on June 29, 2012. Structured case report form questionnaires were designed to record basic demographic information including age, height, weight, body mass index (BMI), smoking/drinking status, and physiological parameters including $\mathrm{HR}$, pulse oxygen saturation $\left(\mathrm{SpO}_{2}\right)$, systolic blood pressure (SBP), and diastolic blood pressure (DBP). Distribution and completion of questionnaires were performed 7 days before the flight and within 18 to 24 hours after arrival at Lhasa $(3700 \mathrm{~m})$, respectively. HAH scores and physiological parameters were obtained in the next morning after arrival. HAH was diagnosed based on the criteria of International Classification of Headache Disorders: second edition, and the characteristics of headache need to meet at least two out of the five criteria: (1) bilateral, (2) frontal or frontotemporal, (3) dull or pressing pain, (4) mild to moderate intensity, and (5) aggravated by exertion, movement, straining, coughing, or bending down $[4,6]$. Due to the difficult condition of field trial and clinical research, HAH scores were classified as follows: 0 for no headache, 1 for mild headache, 2 for moderate headache, and 3 for severe headache [16-18]. Individuals with $\mathrm{HAH}$ score $\geq 1$ were assigned to the $\mathrm{HAH}$ group, while those with $\mathrm{HAH}$ score $=0$ were classified into the non-HAH group. Physiological parameters were measured when participants had rested in a sitting position for more than 10 minutes. $\mathrm{HR}$ and $\mathrm{BP}$ were measured using a 
wrist sphygmomanometer (HEM-6200, OMRON Healthcare Ltd., Kyoto, Japan), while $\mathrm{SpO}_{2}$ was measured using a pulse oximeter (NONIN-9550, Nonin Onyx, Plymouth, MN, USA).

2.3. SNPs Selection and Genotyping. We selected 35 SNPs in EPAS1, HIF1A, HIF1AN, EGLN1, and PPARA from the dbSNP database (http://www.ncbi.nlm.nih.gov/projects/ $\mathrm{SNP} /$ ), and several of them had been reported to be associated with high-altitude illness or hypoxia in previous literatures $[29,30,33]$. SNPs that participated in adaptability adjustment of hypoxia located in noncoding regions more frequently than in coding regions, so we chose SNPs from introns, $3^{\prime}$ or $5^{\prime}$ untranslated regions, and upstream or downstream region of target genes. SNPs were excluded from further association analyses if minor allele frequency (MAF) was less than $5 \%$ or inconsistent with Hardy-Weinberg equilibrium (HWE).

Seven days before the trip, all subjects were requested to fast for 10-12 hours the night before, and we collected $5 \mathrm{ml}$ peripheral venous blood by using $\mathrm{K}_{2}$-EDTA anticoagulant tubes from each subject on the next morning. After centrifugation, we extracted genomic DNA from blood cells by using the Ezup Column Blood Genomic DNA Extraction kit (Shanghai Sangon Biotechnology Co., Ltd., Shanghai, China) according to the manufacturer's instructions, and genomic DNA was stored at $-20^{\circ} \mathrm{C}$ until analysis. We designed the polymerase chain reaction (PCR) primers with Sequenom MassARRAY Assay Design software (version 3.1; Sequenom, Inc., San Diego, CA, USA) and synthesized primers with Shanghai Sangon Biotechnology Co., Ltd (Shanghai, China). MALDI-TOF Mass Spectrometer (Sequenom, Inc., San Diego, CA, USA) was used for genotyping. Sequencing was performed in a blinded fashion, and $10 \%$ of DNA samples were repeatedly genotyped to guarantee the accuracy of results.

2.4. Statistical Analyses. All hypothesis testing analyses were two-sided, and a $P$ value of $<0.05$ was considered as significant. Continuous variables were expressed as mean\pm standard deviation, and differences between HAH group and non-HAH group were analyzed by independent samples $t$-test, while changes of physiological measurements after high-altitude exposure were analyzed by paired samples $t$ test. Categorical variables including smoking/drinking status were expressed as cases and percentages, and differences between groups were analyzed by the chi-square test. Allelic frequencies were calculated from the genotypes. Chi-square test was used to compare allele/genotype frequencies between groups and confirm that distributions of allele/genotype were in accordance with HWE. Binary logistic regression was used to analyze the association between variant genotypes and the risk of developing $\mathrm{HAH}$ under dominant and recessive models, and results were adjusted for potential confounders including age, BMI, smoking/ drinking status, $\mathrm{HR}, \mathrm{SpO}_{2}, \mathrm{SBP}$, and $\mathrm{DBP}$. Odds ratio (OR) and its approximate $95 \%$ confidence interval (CI) were calculated. Associations between gene variants and $\mathrm{HAH}$ intensity were analyzed using the Chi-squared test, by comparing mild HAH group and moderate-severe HAH group with the non-HAH group, respectively. Analyses of linkage disequilibrium (LD) and LD plot were performed by using Haploview 4.2 software. Microsoft Excel, SPSS 24.0 (SPSS Inc., Chicago, IL, USA), and SNPstats (https://www. snpstats.net) online software were also used for data processes and analyses.

\section{Results}

3.1. Characteristics of the Study Population. The study enrolled 580 healthy Chinese Han males, and the mean age was $23.04 \pm 3.71$ years. The characteristics of the study subjects are shown in Table 1 . The incidence of HAH was $72.59 \%$, and there were no cases of HACE or HAPE in this study. No significant difference was noted in the age, height, weight, $\mathrm{BMI}$, drinking status, and baseline physiological parameters between the HAH group and the non-HAH group. However, smoking status significantly differed $(51.07 \%$ in $\mathrm{HAH}$ group vs. $60.38 \%$ in non-HAH group, $P=0.045$ ). As for the physiological measurements at $3700 \mathrm{~m}$, there were significantly higher $\mathrm{HR}(P=0.016)$ and lower $\mathrm{SpO}_{2}(P=0.007)$ in $\mathrm{HAH}$ patients. In general, subjects reported increased HR, SBP, DBP, and decreased $\mathrm{SpO}_{2}$ after high-altitude exposure $(P<0.001)$.

\subsection{Associations between EPAS1/PPARA and HAH.} Detailed information about target SNPs including allele, gene, chromosome position, MAF, HWE test, and genotypic distribution in 580 subjects is presented in Table 2. The results of the HWE test for all target SNPs were $>0.05$. In our study, rs4953354 and rs6756667 in EPAS1 and rs6520015 in PPARA were found to be significantly associated with the risk of developing $\mathrm{HAH}$.

Information of allelic distribution and association analyses between SNPs in EPAS1 and HAH under multiple models is summarized in Table 3. The distribution of rs4953354 "A" allele (HAH, 89.0\% vs. non-HAH, 83.0\%) and "G" allele (HAH, $11.0 \%$ vs. non-HAH, $17.0 \%)$ was significantly different $(P=0.007)$, implying that individuals carrying "G" allele were less susceptible to HAH compared with those who carry " $\mathrm{A}$ " allele. Under the codominant model, the genotypes "AG" $(\mathrm{OR}=0.62 ; 95 \% \mathrm{CI}=0.41-0.95)$ and "GG" (OR $=0.25 ; 95 \% \mathrm{CI}=0.05-1.12)$ were associated with decreased HAH risk $(P=0.023)$. Under the dominant model, the "AG/GG" genotype was significantly associated with decreased risk of HAH (OR $=0.59,95 \% \mathrm{CI}=0.39-0.89$, $P=0.013)$ compared with the "AA" genotype. By comparing the allelic frequency of rs6756667 between two groups, we found that " $\mathrm{A}$ " allele (HAH, $10.0 \%$ vs. non- $\mathrm{HAH}$, $15.1 \%)$ was significantly associated with decreased risk of HAH compared with "G" allele (HAH, $90.0 \%$ vs. non-HAH, 84.9\%; $P=0.014$ ). Under the dominant model, the "AG/ AA" genotype was significantly associated with decreased risk of HAH $(\mathrm{OR}=0.63,95 \% \mathrm{CI}=0.41-0.97, P=0.036)$ compared with the "AA" genotype. The results above remained significant after adjustment for multiple factors. 
TABLE 1: Basic characteristics of subjects.

\begin{tabular}{|c|c|c|c|c|c|c|c|c|c|}
\hline \multirow[b]{2}{*}{ Variables } & \multicolumn{4}{|c|}{ Baseline measurements } & \multicolumn{5}{|c|}{ Measurements at $3700 \mathrm{~m}$} \\
\hline & Total (580) & $\mathrm{HAH}+(421)$ & HAH- (159) & $\begin{array}{c}P \\
\text { value }^{\mathrm{a}}\end{array}$ & Total (580) & $\mathrm{HAH}+(421)$ & HAH- (159) & $\begin{array}{c}P \\
\text { value }^{\mathrm{a}}\end{array}$ & $P$ value ${ }^{\#}$ \\
\hline \multicolumn{10}{|c|}{ Basic demographic data } \\
\hline Age (year) & $23.04 \pm 3.71$ & $23.22 \pm 3.89$ & $22.58 \pm 3.12$ & 0.067 & $23.04 \pm 3.71$ & $23.22 \pm 3.89$ & $22.58 \pm 3.12$ & 0.067 & - \\
\hline Height $(\mathrm{cm})$ & $171.49 \pm 4.64$ & $171.42 \pm 4.64$ & $171.67 \pm 4.64$ & 0.571 & $171.49 \pm 4.64$ & $171.42 \pm 4.64$ & $171.67 \pm 4.64$ & 0.571 & - \\
\hline Weight (kg) & $64.09 \pm 7.42$ & $64.19 \pm 7.74$ & $63.84 \pm 6.50$ & 0.615 & $64.09 \pm 7.42$ & $64.19 \pm 7.74$ & $63.84 \pm 6.50$ & 0.615 & - \\
\hline BMI $\left(\mathrm{kg} / \mathrm{m}^{2}\right)$ & $21.78 \pm 2.20$ & $21.82 \pm 2.29$ & $21.65 \pm 1.94$ & 0.410 & $21.78 \pm 2.20$ & $21.82 \pm 2.29$ & $21.65 \pm 1.94$ & 0.410 & - \\
\hline $\begin{array}{l}\text { Current } \\
\text { smokers }\end{array}$ & $311(53.62)$ & $215(51.07)$ & $96(60.38)$ & $0.045^{\mathrm{b} *}$ & $311(53.62)$ & $215(51.07)$ & $96(60.38)$ & $0.045^{\mathrm{b} *}$ & - \\
\hline $\begin{array}{l}\text { Current } \\
\text { drinkers }\end{array}$ & $25(4.31)$ & $15(3.56)$ & $10(6.29)$ & $0.149^{\mathrm{b}}$ & $25(4.31)$ & $15(3.56)$ & $10(6.29)$ & $0.149^{\mathrm{b}}$ & - \\
\hline \multicolumn{10}{|c|}{ Physiological parameters } \\
\hline $\mathrm{HR}(\mathrm{bpm})$ & $65.17 \pm 10.39$ & $65.17 \pm 10.63$ & $65.17 \pm 9.78$ & 0.997 & $85.53 \pm 12.97$ & $86.33 \pm 13.44$ & $83.43 \pm 11.42$ & $0.016^{*}$ & $<0.001^{*}$ \\
\hline $\mathrm{SpO}_{2}(\%)$ & $98.14 \pm 1.00$ & $98.13 \pm 1.02$ & $98.19 \pm 0.95$ & 0.562 & $88.47 \pm 3.14$ & $88.25 \pm 3.20$ & $89.04 \pm 2.91$ & $0.007^{*}$ & $<0.001^{*}$ \\
\hline $\mathrm{SBP}(\mathrm{mmHg})$ & $115.89 \pm 10.83$ & $115.91 \pm 10.85$ & $115.83 \pm 10.82$ & 0.945 & $118.39 \pm 11.41$ & $118.74 \pm 11.85$ & $117.45 \pm 10.13$ & 0.225 & $<0.001^{*}$ \\
\hline $\begin{array}{l}\text { DBP } \\
(\mathrm{mmHg})\end{array}$ & $73.81 \pm 9.48$ & $74.25 \pm 9.76$ & $72.64 \pm 8.60$ & 0.117 & $78.78 \pm 9.83$ & $79.23 \pm 10.01$ & $77.61 \pm 9.26$ & 0.077 & $<0.001^{*}$ \\
\hline
\end{tabular}

TABLE 2: SNP information and genotype distribution in 580 subjects.

\begin{tabular}{|c|c|c|c|c|c|c|c|}
\hline SNP & Allele & Gene & Chromosome position & $N$ & Genotype frequencies & MAF (\%) & $P$ value for HWE test \\
\hline rs13419896 & $\mathrm{G} / \mathrm{A}$ & EPAS1 & Chr2:46329206 & 577 & $0.47 / 0.43 / 0.10$ & 31.0 & 1.000 \\
\hline rs4953354 & $\mathrm{A} / \mathrm{G}$ & EPAS1 & Chr2:46348249 & 580 & $0.76 / 0.23 / 0.01$ & 13.0 & 0.460 \\
\hline rs6756667 & G/A & EPAS1 & Chr2:46352270 & 580 & $0.78 / 0.21 / 0.01$ & 11.0 & 0.680 \\
\hline rs7292407 & $\mathrm{C} / \mathrm{A}$ & PPARA & Chr22:46057832 & 549 & $0.74 / 0.23 / 0.03$ & 15.0 & 0.090 \\
\hline rs6520015 & $\mathrm{T} / \mathrm{C}$ & PPARA & Chr22:46067551 & 580 & $0.69 / 0.28 / 0.03$ & 17.0 & 0.770 \\
\hline rs 4253623 & $\mathrm{~A} / \mathrm{G}$ & PPARA & Chr22:46154203 & 575 & $0.75 / 0.23 / 0.02$ & 13.0 & 1.000 \\
\hline rs 135538 & $\mathrm{G} / \mathrm{C}$ & PPARA & Chr22:46168728 & 577 & $0.33 / 0.46 / 0.21$ & 44.0 & 0.180 \\
\hline rs4253681 & $\mathrm{T} / \mathrm{C}$ & PPARA & Chr22:46183703 & 579 & $0.64 / 0.32 / 0.04$ & 20.0 & 0.890 \\
\hline rs4253747 & $\mathrm{T} / \mathrm{A}$ & PPARA & Chr22:46217340 & 580 & $0.63 / 0.33 / 0.04$ & 27.0 & 0.900 \\
\hline rs2009873 & $\mathrm{A} / \mathrm{G}$ & EGLN1 & Chr1:231363490 & 576 & $0.34 / 0.48 / 0.18$ & 42.0 & 0.550 \\
\hline rs 2066140 & $\mathrm{G} / \mathrm{C}$ & EGLN1 & Chr1:231368565 & 578 & $0.34 / 0.48 / 0.18$ & 42.0 & 0.800 \\
\hline rs2739513 & $\mathrm{A} / \mathrm{G}$ & EGLN1 & Chr1:231379455 & 563 & $0.34 / 0.48 / 0.18$ & 42.0 & 0.600 \\
\hline rs 2486736 & $\mathrm{~A} / \mathrm{G}$ & EGLN1 & Chr1:231385732 & 572 & $0.33 / 0.49 / 0.18$ & 42.0 & 1.000 \\
\hline rs480902 & $\mathrm{C} / \mathrm{T}$ & EGLN1 & Chr1:231395881 & 579 & $0.34 / 0.48 / 0.18$ & 42.0 & 0.860 \\
\hline rs508618 & $\mathrm{A} / \mathrm{G}$ & EGLN1 & Chr1:231396566 & 578 & $0.78 / 0.21 / 0.01$ & 11.0 & 0.130 \\
\hline rs2790882 & $\mathrm{A} / \mathrm{G}$ & EGLN1 & Chr1:231397037 & 575 & $0.33 / 0.49 / 0.18$ & 43.0 & 0.930 \\
\hline rs2486729 & $\mathrm{A} / \mathrm{G}$ & EGLN1 & Chr1:231399838 & 576 & $0.34 / 0.45 / 0.20$ & 43.0 & 0.089 \\
\hline rs7542797 & $\mathrm{A} / \mathrm{C}$ & EGLN1 & Chr1:231418041 & 577 & $0.76 / 0.22 / 0.01$ & 12.0 & 0.850 \\
\hline rs1339891 & G/A & EGLN1 & Chr1:231420649 & 579 & $0.81 / 0.18 / 0.01$ & 10.0 & 1.000 \\
\hline rs12406290 & $\mathrm{A} / \mathrm{G}$ & EGLN1 & Chr1:231423480 & 561 & $0.28 / 0.49 / 0.22$ & 47.0 & 0.870 \\
\hline rs2153364 & $\mathrm{A} / \mathrm{G}$ & EGLN1 & Chr1:231424474 & 530 & $0.28 / 0.49 / 0.23$ & 48.0 & 0.073 \\
\hline rs 2275279 & $\mathrm{~A} / \mathrm{T}$ & EGLN1 & Chr1:231591348 & 579 & $0.54 / 0.38 / 0.08$ & 27.0 & 0.250 \\
\hline rs2301104 & $\mathrm{G} / \mathrm{C}$ & HIF1A & Chr14:61698310 & 579 & $0.87 / 0.13 / 0.00$ & 7.0 & 0.500 \\
\hline rs12434438 & $\mathrm{A} / \mathrm{G}$ & HIF1A & Chr14:61730580 & 568 & $0.56 / 0.39 / 0.05$ & 24.0 & 0.170 \\
\hline rs966824 & $\mathrm{C} / \mathrm{T}$ & HIF1A & Chr14:61733800 & 574 & $0.68 / 0.30 / 0.02$ & 17.0 & 0.380 \\
\hline rs2301112 & $\mathrm{A} / \mathrm{C}$ & HIF1A & Chr14:61739455 & 545 & $0.91 / 0.09 / 0.00$ & 5.0 & 1.000 \\
\hline rs2301113 & $\mathrm{A} / \mathrm{C}$ & HIF1A & Chr14:61739830 & 576 & $0.43 / 0.46 / 0.10$ & 34.0 & 0.400 \\
\hline rs2295778 & $\mathrm{C} / \mathrm{G}$ & HIF1AN & Chr10:100536079 & 578 & $0.58 / 0.36 / 0.06$ & 24.0 & 0.910 \\
\hline rs11190602 & $\mathrm{T} / \mathrm{C}$ & HIF1AN & Chr10:100537499 & 578 & $0.78 / 0.20 / 0.02$ & 12.0 & 0.420 \\
\hline rs3750633 & $\mathrm{G} / \mathrm{A}$ & HIF1AN & Chr10:100548457 & 579 & $0.85 / 0.14 / 0.01$ & 8.0 & 0.390 \\
\hline rs10883512 & $\mathrm{A} / \mathrm{G}$ & HIF1AN & Chr10:100548759 & 579 & $0.85 / 0.14 / 0.01$ & 8.0 & 0.560 \\
\hline rs11816840 & $\mathrm{G} / \mathrm{C}$ & HIF1AN & Chr10:100549463 & 579 & $0.85 / 0.14 / 0.01$ & 8.0 & 0.770 \\
\hline rs1054399 & $\mathrm{C} / \mathrm{T}$ & HIF1AN & Chr10:100552808 & 579 & $0.85 / 0.14 / 0.01$ & 8.0 & 0.390 \\
\hline rs11292 & $\mathrm{T} / \mathrm{C}$ & HIF1AN & Chr10:100553850 & 580 & $0.85 / 0.14 / 0.01$ & 8.0 & 0.390 \\
\hline rs11190613 & $\mathrm{T} / \mathrm{C}$ & HIF1AN & Chr10:100554240 & 580 & $0.85 / 0.14 / 0.01$ & 8.0 & 0.390 \\
\hline
\end{tabular}

SNP, single nucleotide polymorphism; MAF, minor allele frequency; HWE, Hardy-Weinberg equilibrium. 
TABLE 3: Association between SNPs in EPAS1 and HAH under multiple genetic models.

\begin{tabular}{|c|c|c|c|c|c|c|c|c|}
\hline SNP & Model & Allele/genotype & $\mathrm{HAH}+[n(\%)]$ & HAH- $[n(\%)]$ & OR $(95 \% \mathrm{CI})$ & $P$ value & OR $(95 \% \mathrm{CI})^{\mathrm{a}}$ & $P$ value $^{\mathrm{a}}$ \\
\hline \multirow{9}{*}{ rs4953354 } & \multirow{2}{*}{ Allele } & $\mathrm{A}$ & $749(89.0)$ & $264(83.0)$ & & \multirow[t]{2}{*}{$0.007^{*}$} & & \\
\hline & & G & $93(11.0)$ & $54(17.0)$ & & & & \\
\hline & \multirow{3}{*}{ Codominant } & $\mathrm{AA}$ & $331(78.6)$ & $109(68.5)$ & 1 & \multirow[t]{3}{*}{$0.023^{*}$} & 1 & \multirow[t]{3}{*}{$0.046^{*}$} \\
\hline & & AG & $87(20.7)$ & $46(28.9)$ & $0.62(0.41-0.95)$ & & $0.61(0.40-0.94)$ & \\
\hline & & GG & $3(0.7)$ & $4(2.5)$ & $0.25(0.05-1.12)$ & & $0.37(0.08-1.74)$ & \\
\hline & \multirow{2}{*}{ Dominant } & AA & $331(78.6)$ & $109(68.5)$ & 1 & \multirow[t]{2}{*}{$0.013^{*}$} & 1 & \multirow{2}{*}{$0.016^{*}$} \\
\hline & & AG/GG & $90(21.4)$ & $50(31.4)$ & $0.59(0.39-0.89)$ & & $0.59(0.39-0.90)$ & \\
\hline & \multirow{2}{*}{ Recessive } & $\mathrm{AA} / \mathrm{AG}$ & $418(99.3)$ & $155(97.5)$ & 1 & \multirow[t]{2}{*}{0.097} & 1 & \multirow[t]{2}{*}{0.250} \\
\hline & & GG & $3(0.7)$ & $4(2.5)$ & $0.28(0.06-1.26)$ & & $0.41(0.09-1.93)$ & \\
\hline \multirow{9}{*}{ rs6756667 } & \multirow{2}{*}{ Allele } & $\mathrm{G}$ & $758(90.0)$ & $270(84.9)$ & & \multirow[t]{2}{*}{$0.014^{*}$} & & \multirow{5}{*}{0.061} \\
\hline & & A & $84(10.0)$ & $48(15.1)$ & & & & \\
\hline & \multirow{3}{*}{ Codominant } & GG & $339(80.5)$ & $115(72.3)$ & 1 & \multirow[t]{3}{*}{$0.030^{*}$} & 1 & \\
\hline & & AG & $80(19)$ & $40(25.2)$ & $0.68(0.44-1.05)$ & & $0.67(0.43-1.05)$ & \\
\hline & & AA & $2(0.5)$ & $4(2.5)$ & $0.17(0.03-0.94)$ & & $0.23(0.04-1.29)$ & \\
\hline & \multirow{2}{*}{ Dominant } & GG & $339(80.5)$ & $115(72.3)$ & 1 & \multirow[t]{2}{*}{$0.036^{*}$} & 1 & \multirow[t]{2}{*}{$0.044^{*}$} \\
\hline & & AG/AA & $82(19.5)$ & $44(27.7)$ & $0.63(0.41-0.97)$ & & $0.64(0.41-0.98)$ & \\
\hline & \multirow{2}{*}{ Recessive } & GG/AG & $419(99.5)$ & $155(97.5)$ & 1 & \multirow[t]{2}{*}{0.044} & 1 & \multirow[t]{2}{*}{0.100} \\
\hline & & AA & $2(0.5)$ & $4(2.5)$ & $0.18(0.03-1.02)$ & & $0.25(0.04-1.41)$ & \\
\hline
\end{tabular}

Allelic frequencies were compared by the Chi-squared test $(2 * 2$ contingency table, $\mathrm{df}=1)$. Association between SNPs and HAH under different models was detected by using binary logistic regression. a Adjusted for age, height, weight, BMI, smoking and drinking status, $\mathrm{HR}, \mathrm{SpO} \mathrm{O}_{2}, \mathrm{SBP}$, and $\mathrm{DBP}$. ${ }^{*} P<0.05$ indicated statistical significance. $\mathrm{HAH}+$, subjects with $\mathrm{HAH}$; HAH-, subjects without $\mathrm{HAH}$. OR, odds ratio; 95\% CI, 95\% confidence interval; SNP, single nucleotide polymorphism; $\mathrm{HAH}$, high-altitude headache; $\mathrm{BMI}$, body mass index; $\mathrm{HR}$, heart rate; $\mathrm{SpO}_{2}$, pulse oxygen saturation; $\mathrm{SBP}$, systolic blood pressure; DBP, diastolic blood pressure.

However, no statistically significant association was found for rs4953354 or rs6756667 under the recessive model.

As summarized in Table 4, the distribution of rs6520015 "T" allele (HAH, 84.4\% vs. non-HAH, 78.3\%) and "C" allele (HAH, $15.6 \%$ vs. non-HAH, $21.7 \%, P=0.014$ ) was significantly different, suggesting a dominant effect of "C" allele in lowering the HAH risk. Under the dominant model, the proportion of non-HAH subjects with at least one "C" allele was much higher than $\mathrm{HAH}$ patients $(\mathrm{OR}=0.60,95 \%$ $\mathrm{CI}=0.41-0.88, P=0.009$ ), and results remained significant after multivariable calibration. However, no association was found under the codominant model or recessive model. The "CA/AA" genotype of rs7292407 was associated with lower $\mathrm{HAH}$ risk $(\mathrm{OR}=0.65,95 \% \mathrm{CI}=0.43-0.98, \quad P=0.041)$ compared with the "CC" genotype under dominant model; however, results turned insignificant after adjustment for multiple factors. No significant difference was found in the allelic distribution, under the codominant model or recessive model between the HAH group and non-HAH group.

No association was found between HIF1A/HIF1AN/ EGLN1 and HAH under the dominant model. Detailed information of association analyses between other SNPs and HAH under multiple models was summarized in Supplement Table 1 .

3.3. Associations between EPAS1/PPARA and HAH Intensity. Subjects were divided into four groups according to HAH scores: $0=$ non-HAH, $1=$ mild HAH, $2=$ moderate $\mathrm{HAH}$, and $3=$ severe HAH. The sample size of severe HAH was much smaller, so we combined it together with moderate $\mathrm{HAH}$ into one group when comparing allelic frequencies and genotypic distributions between groups. We found that rs4953354 and rs6520015 were associated with
HAH intensity. As shown in Table 5, the distribution of rs4953354 "G" allele (mild HAH, $11.5 \%$ vs. non-HAH, $17.0 \%, P=0.017$; moderate-severe $\mathrm{HAH}, 9.4 \%$ vs. nonHAH, $17.0 \%, P=0.023$ ) was significantly different between groups. Under the dominant model, the "AG/GG" genotype of rs4953354 was significantly associated with decreased risk of mild HAH (mild HAH, $22.0 \%$ vs. non-HAH, 31.4\%; $P=0.024$ ) and moderate-severe HAH (moderate-severe $\mathrm{HAH}, 18.8 \%$ vs. non- $\mathrm{HAH}, 31.4 \%$; $P=0.034)$. The distribution of rs6520015 "C" allele (mild HAH, $15.9 \%$ vs. non$\mathrm{HAH}, 21.7 \%, P=0.026$; moderate-severe $\mathrm{HAH}, 14.1 \%$ vs. non-HAH, $21.7 \%, P=0.042$ ) was significantly different between groups. Under the dominant model, the "CT/CC" genotype of rs6520015 was significantly associated with decreased risk of mild HAH (mild HAH, $28.3 \%$ vs. nonHAH, 39.6\%; $P=0.011$ ). No significant association was found between rs6520015 and moderate-severe HAH, and rs6756667 exhibited no significant difference in the development of mild HAH or moderate-severe HAH.

3.4. LD Analyses and Association between PPARA Haplotypes and HAH. The mutants of EPAS1 and PPARA have been proved to be associated with $\mathrm{HAH}$ after acute high-altitude exposure. The loci of rs4953354 and rs6756667 located in the same gene, and the position of rs6520015 on the chromosome was close to rs7292407, rs4253623, rs135538, rs4253681, and rs4253747, so we drew linkage map of EPAS1 and PPARA, respectively, using Haploview. As shown in Figure 1, two LD blocks were found in PPARA. Block 1 consisted of rs7292407 and rs6520015, and values of $\mathrm{D}^{\prime}, r^{2}$, and LOD in Block 1 were 0.985, 0.812, and 121.37, respectively. Three common haplotypes (frequencies $\geq 1 \%$ ) of Block 1 are exhibited in Table 6. Carriers of the 
TABLE 4: Association between SNPs in PPARA and HAH under multiple genetic models.

\begin{tabular}{|c|c|c|c|c|c|c|c|c|}
\hline SNP & Model & Allele/genotype & $\mathrm{HAH}+[n(\%)]$ & HAH- $[n(\%)]$ & OR $(95 \% \mathrm{CI})$ & $P$ value & OR $(95 \% C I)^{a}$ & $P$ value $^{\mathrm{a}}$ \\
\hline \multirow{9}{*}{ rs7292407 } & \multirow{2}{*}{ Allele } & $\mathrm{C}$ & $685(86.5)$ & $251(82.0)$ & & \multirow[t]{2}{*}{0.061} & & \multirow{5}{*}{0.150} \\
\hline & & A & $107(13.5)$ & $55(18.0)$ & & & & \\
\hline & \multirow{3}{*}{ Codominant } & $\mathrm{CC}$ & $301(76.0)$ & $103(67.3)$ & 1 & \multirow[t]{3}{*}{0.110} & 1 & \\
\hline & & $\mathrm{CA}$ & $83(21.0)$ & $45(29.4)$ & $0.63(0.41-0.97)$ & & $0.64(0.42-1.00)$ & \\
\hline & & AA & $12(3.0)$ & $5(3.3)$ & $0.82(0.28-2.39)$ & & $0.93(0.31-2.79)$ & \\
\hline & \multirow{2}{*}{ Dominant } & $\mathrm{CC}$ & $301(76.0)$ & $103(67.3)$ & 1 & \multirow[t]{2}{*}{$0.041^{*}$} & 1 & \multirow[t]{2}{*}{0.066} \\
\hline & & CA/AA & $95(24.0)$ & $50(32.7)$ & $0.65(0.43-0.98)$ & & $0.67(0.44-1.02)$ & \\
\hline & \multirow{2}{*}{ Recessive } & $\mathrm{CC} / \mathrm{CA}$ & $384(97.0)$ & $148(96.7)$ & 1 & \multirow[t]{2}{*}{0.890} & 1 & \multirow[t]{2}{*}{0.930} \\
\hline & & AA & $12(3.0)$ & $5(3.3)$ & $0.93(0.32-2.67)$ & & $1.05(0.35-3.11)$ & \\
\hline \multirow{9}{*}{ rs6520015 } & \multirow{2}{*}{ Allele } & $\mathrm{T}$ & $711(84.4)$ & $249(78.3)$ & & \multirow[t]{2}{*}{$0.014^{*}$} & & \\
\hline & & $\mathrm{C}$ & $131(15.6)$ & $69(21.7)$ & & & & \\
\hline & \multirow{3}{*}{ Codominant } & TT & $302(71.7)$ & $96(60.4)$ & 1 & \multirow[t]{3}{*}{$0.034^{*}$} & 1 & \multirow[t]{3}{*}{0.059} \\
\hline & & CT & $107(25.4)$ & $57(35.9)$ & $0.60(0.40-0.89)$ & & $0.61(0.41-0.92)$ & \\
\hline & & $\mathrm{CC}$ & $12(2.8)$ & $6(3.8)$ & $0.64(0.23-1.74)$ & & $0.72(0.25-2.02)$ & \\
\hline & \multirow{2}{*}{ Dominant } & TT & $302(71.7)$ & $96(60.4)$ & 1 & \multirow[t]{2}{*}{$0.009^{*}$} & 1 & \multirow[t]{2}{*}{$0.018^{*}$} \\
\hline & & $\mathrm{CT} / \mathrm{CC}$ & $119(28.3)$ & $63(39.6)$ & $0.60(0.41-0.88)$ & & $0.62(0.42-0.92)$ & \\
\hline & \multirow{2}{*}{ Recessive } & $\mathrm{TT} / \mathrm{CT}$ & $409(97.2)$ & $153(96.2)$ & 1 & \multirow[t]{2}{*}{0.570} & 1 & \multirow[t]{2}{*}{0.750} \\
\hline & & $\mathrm{CC}$ & $12(2.8)$ & $6(3.8)$ & $0.75(0.28-2.03)$ & & $0.84(0.30-2.35)$ & \\
\hline
\end{tabular}

Allelic frequencies were compared by Chi-squared test $\left(2^{*} 2\right.$ contingency table, $\left.\mathrm{df}=1\right)$. Association between SNPs and HAH under different models was detected by using binary logistic regression. ${ }^{a}$ Adjusted for age, height, weight, BMI, smoking and drinking status, $\mathrm{HR}, \mathrm{SpO}_{2}, \mathrm{SBP}$, and $\mathrm{DBP}$. ${ }^{*} P<0.05$ indicated statistical significance. HAH+, subjects with $\mathrm{HAH}$; HAH-, subjects without HAH. See Table 3 for group abbreviations.

TABLE 5: Association between rs4953354, rs6756667, rs6520015, and HAH intensity.

\begin{tabular}{|c|c|c|c|c|c|c|c|}
\hline SNP & Model & Allele/genotype & non-HAH $[n(\%)]$ & Mild HAH $[n(\%)]$ & Moderate-severe $\mathrm{HAH}[n(\%)]$ & $P$ value $^{\mathrm{a}}$ & $P$ value $^{\mathrm{b}}$ \\
\hline \multirow{6}{*}{ rs4953354 } & \multirow{2}{*}{ Allele } & $\mathrm{A}$ & $264(83.0)$ & $595(88.5)$ & $154(90.6)$ & \multirow[t]{2}{*}{$0.017^{*}$} & \multirow[t]{2}{*}{$0.023^{*}$} \\
\hline & & G & $54(17.0)$ & $77(11.5)$ & $16(9.4)$ & & \\
\hline & \multirow{2}{*}{ Dominant } & AA & $109(68.6)$ & $262(78.0)$ & $69(81.2)$ & \multirow[t]{2}{*}{$0.024^{*}$} & \multirow[t]{2}{*}{$0.034^{*}$} \\
\hline & & AG/GG & $50(31.4)$ & $74(22.0)$ & $16(18.8)$ & & \\
\hline & \multirow{2}{*}{ Recessive } & $\mathrm{AA} / \mathrm{AG}$ & $155(97.5)$ & $333(99.1)$ & $85(100.0)$ & \multirow[t]{2}{*}{0.219} & \multirow[t]{2}{*}{0.301} \\
\hline & & GG & $4(2.5)$ & $3(0.9)$ & $0(0.0)$ & & \\
\hline \multirow{6}{*}{ rs6756667 } & \multirow{2}{*}{ Allele } & $\mathrm{G}$ & $270(84.9)$ & $603(89.7)$ & $155(91.2)$ & \multirow[t]{2}{*}{$0.028^{*}$} & \multirow[t]{2}{*}{$0.049^{*}$} \\
\hline & & $\mathrm{A}$ & $48(15.1)$ & $69(10.3)$ & $15(8.8)$ & & \\
\hline & \multirow[b]{2}{*}{ Dominant } & GG & $115(72.3)$ & $269(80.1)$ & $70(82.4)$ & \multirow[t]{2}{*}{0.054} & \multirow[t]{2}{*}{0.081} \\
\hline & & AG/AA & $44(27.7)$ & $67(19.9)$ & 15 (17.6) & & \\
\hline & \multirow{2}{*}{ Recessive } & GG/AG & $155(97.5)$ & $334(99.4)$ & $85(100.0)$ & \multirow[t]{2}{*}{0.087} & \multirow[t]{2}{*}{0.301} \\
\hline & & AA & $4(2.5)$ & $2(0.6)$ & $0(0.0)$ & & \\
\hline \multirow{6}{*}{ rs6520015 } & \multirow{2}{*}{ Allele } & $\mathrm{T}$ & $249(78.3)$ & $565(84.1)$ & $146(85.9)$ & \multirow[t]{2}{*}{$0.026^{*}$} & \multirow[t]{2}{*}{$0.042^{*}$} \\
\hline & & $\mathrm{C}$ & $69(21.7)$ & 107 (15.9) & $24(14.1)$ & & \\
\hline & \multirow{2}{*}{ Dominant } & $\mathrm{TT}$ & $96(60.4)$ & $241(71.7)$ & $61(71.8)$ & \multirow[t]{2}{*}{$0.011^{*}$} & \multirow[t]{2}{*}{0.077} \\
\hline & & CT/CC & $63(39.6)$ & $95(28.3)$ & $24(28.2)$ & & \\
\hline & \multirow{2}{*}{ Recessive } & TT/CT & $153(96.2)$ & $324(96.4)$ & $85(100.0)$ & \multirow[t]{2}{*}{0.911} & \multirow[t]{2}{*}{0.095} \\
\hline & & $\mathrm{CC}$ & $6(3.8)$ & $12(3.6)$ & $0(0.0)$ & & \\
\hline
\end{tabular}

Association between SNPs and HAH intensity was analyzed in allele-dose, under dominant and recessive model by using Chi-squared test $(2 * 2$ contingency table, $\mathrm{df}=1$ ). ${ }^{\mathrm{a}}$ Mild HAH group vs. non-HAH group; ${ }^{b}$ Moderate-severe HAH group vs. non-HAH group. ${ }^{*} P<0.05$ indicated statistical significance. See Table 3 for group abbreviations.

rs7292407-rs6520015 "C-C" haplotype showed a lower risk of developing $\mathrm{HAH}(\mathrm{OR}=0.41,95 \% \mathrm{CI}=0.19-0.89$, $P=0.024)$, and results remained significant after adjustment for multiple factors. Block 2 contained rs4253623 and rs135538, and values of $\mathrm{D}^{\prime}, r^{2}$, and LOD in Block 2 were $0.966,0.184$, and 29.8, respectively (Figure 1). Three common haplotypes (frequencies $\geq 1 \%$ ) of Block 2 were identified; however, no significant association was observed with HAH risk (Table 6). In addition, we found no haplotype block in EPAS1 (Supplement Figure 1).
3.5. Combined Effects of EPAS1 and PPARA with HAH Risk. Two loci (rs4953354 in EPAS1 and rs6520015 in PPARA) were in association with $\mathrm{HAH}$ especially mild $\mathrm{HAH}$ after acute high-altitude exposure. We wondered whether subjects carrying two genes' mutants would show lower $\mathrm{HAH}$ risk compared with carriers of one mutant or no mutant at all. As rs6756667 played a weak role in the processes of HAH and made no difference in HAH intensity, we did not include it as a grouping criterion. Subjects were divided into 4 subgroups according to the carrying status of gene mutants (see Table 7). Compared with subjects in subgroup 1, 


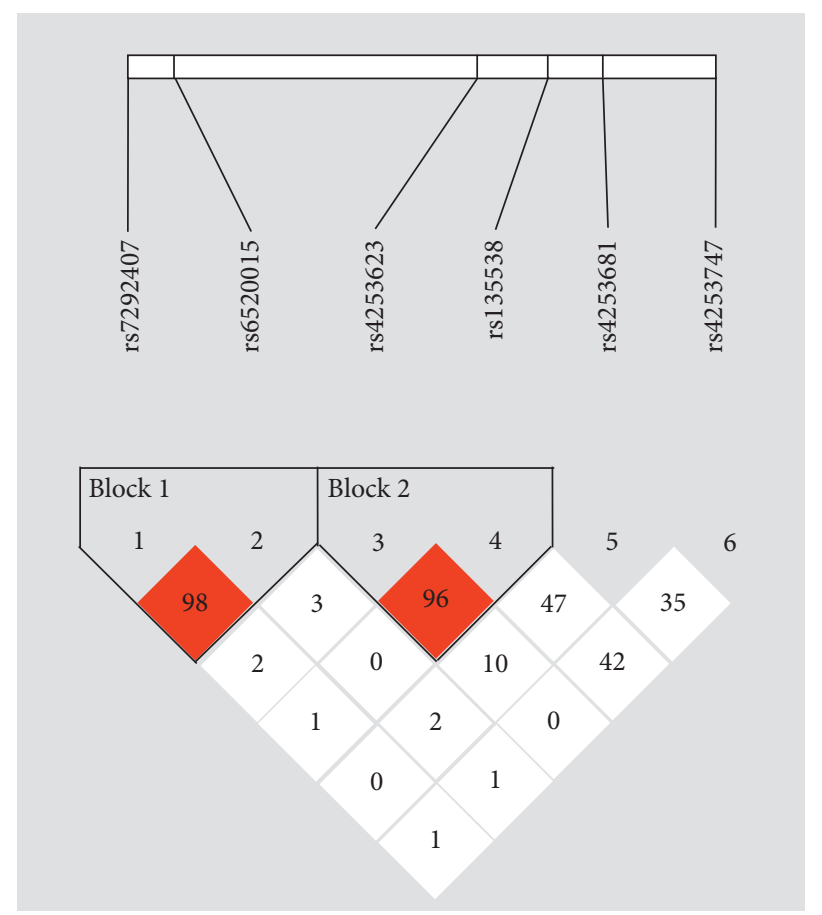

FIGURE 1: Haplotype block map for tag SNPs in PPARA.

TABLE 6: Distributions of PPARA haplotypes and the association with HAH risk.

\begin{tabular}{|c|c|c|c|c|c|c|c|}
\hline \multirow{2}{*}{\multicolumn{2}{|c|}{ Haplotypes }} & \multicolumn{2}{|c|}{ Frequencies } & \multirow[b]{2}{*}{ OR $(95 \% \mathrm{CI})$} & \multirow{2}{*}{$P$ value } & \multirow{2}{*}{ OR $(95 \% \mathrm{CI})^{\mathrm{a}}$} & \multirow{2}{*}{$P$ value } \\
\hline & & $\mathrm{HAH}+$ & HAH- & & & & \\
\hline rs7292407 & rs6520015 & & & & & & \\
\hline $\mathrm{C}$ & $\mathrm{T}$ & 0.783 & 0.826 & 1 & & 1 & \\
\hline A & $\mathrm{C}$ & 0.175 & 0.147 & $0.71(0.50-1.01)$ & 0.057 & $0.74(0.52-1.06)$ & 0.110 \\
\hline $\mathrm{C}$ & $\mathrm{C}$ & 0.042 & 0.026 & $0.41(0.19-0.89)$ & $0.024^{*}$ & $0.41(0.19-0.92)$ & $0.030^{*}$ \\
\hline \multicolumn{8}{|c|}{ Global haplotype association $P$ value: $0.045^{*}$} \\
\hline rs4253623 & rs135538 & & & & & & \\
\hline A & G & 0.552 & 0.565 & 1 & & 1 & \\
\hline A & $\mathrm{C}$ & 0.316 & 0.293 & $1.09(0.83-1.45)$ & 0.530 & $1.14(0.86-1.53)$ & 0.370 \\
\hline G & $\mathrm{C}$ & 0.131 & 0.137 & $0.97(0.66-1.45)$ & 0.900 & $1.02(0.68-1.53)$ & 0.940 \\
\hline
\end{tabular}

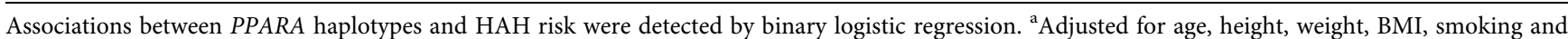
drinking status, $\mathrm{HR}, \mathrm{SpO}_{2}, \mathrm{SBP}$, and DBP. ${ }^{*} P<0.05$ indicated statistical significance. $\mathrm{HAH}+$, subjects with $\mathrm{HAH} ; \mathrm{HAH}-$, subjects without $\mathrm{HAH}$. See Table 3 for group abbreviations.

TABLe 7: Distribution of rs4953354 and rs6520015 in HAH and non-HAH groups.

\begin{tabular}{|c|c|c|c|c|c|c|}
\hline Subgroups & $\mathrm{HAH}+[n(\%)]$ & HAH- $[n(\%)]$ & OR $(95 \% \mathrm{CI})$ & $P$ value & OR $(95 \% \mathrm{CI})^{\mathrm{a}}$ & $P$ value $^{\mathrm{a}}$ \\
\hline 1 & $237(56.29)$ & $66(41.51)$ & 1 & & 1 & \\
\hline 2 & $65(15.44)$ & $30(18.87)$ & $0.60(0.36-1.01)$ & 0.053 & $0.60(0.35-1.02)$ & 0.059 \\
\hline 3 & $94(22.33)$ & $43(27.04)$ & $0.61(0.39-0.96)$ & $0.032^{*}$ & $0.63(0.40-0.99)$ & $0.049^{*}$ \\
\hline 4 & $25(5.94)$ & $20(12.58)$ & $0.35(0.18-0.67)$ & $0.001^{*}$ & $0.36(0.18-0.70)$ & $0.003^{*}$ \\
\hline
\end{tabular}

Subgroup 1: subjects carrying neither rs4953354 "AG/GG” nor rs6520015 “CT/CC"; subgroup 2: subjects carrying rs4953354 "AG/GG” but not rs6520015 "CT/CC"; subgroup 3: subjects carrying rs6520015 "CT/CC" but not rs4953354 "AG/GG"; subgroup 4: subjects carrying both rs4953354 "AG/GG" and rs6520015 "CT/CC". adjusted for age, height, weight, BMI, smoking and drinking status, HR, $\mathrm{SpO}_{2}$, SBP, and DBP. * $P<0.05$ indicated statistical significance. $\mathrm{HAH}+$, subjects with $\mathrm{HAH}$; $\mathrm{HAH}-$, subjects without HAH. See Table 3 for group abbreviations.

subjects in subgroup 3 who carried rs6520015 “CT/CC” were less susceptible to $\mathrm{HAH}(\mathrm{OR}=0.61,95 \% \mathrm{CI}=0.39-0.96$, $P=0.032$ ); however, rs4953354 "AG/GG" carriers showed no significant difference. In the same way, subjects in subgroup 4 who carried both rs4953354 "AG/GG" and rs6520015 "CT/CC" performed larger proportion in the non-HAH group: $12.6 \%$ vs. $5.9 \% \quad$ (OR $=0.35,95 \%$ $\mathrm{CI}=0.18-0.67, P=0.001)$ in comparison with subjects in 
subgroup 1. Results remained significant after multivariable calibration for age, BMI, smoking/drinking status, $\mathrm{HR}$, $\mathrm{SpO}_{2}, \mathrm{SBP}$, and DBP. In a word, EPAS1 and PPARA gene mutants played a synergistic role in decreasing $\mathrm{HAH}$ risk after acute hypoxia exposure.

\section{Discussion}

To the best of our knowledge, we first established the association between EPAS1 and PPARA polymorphisms and the risk of developing $\mathrm{HAH}$ in the Chinese Han population. Subjects carrying rs4953354 "AG/GG” genotype, rs6756667 "AG/AA" genotype, or rs6520015 "CT/CC" genotype were less susceptible to $\mathrm{HAH}$ compared with subjects carrying wild types. To be precise, rs4953354 was associated with decreased risk of mild $\mathrm{HAH}$ and moderate-severe $\mathrm{HAH}$, whereas rs6520015 was associated with reducing the risk of mild HAH. LD phenomenon existed in PPARA, and rs7292407-rs6520015 "C-C" haplotype was associated with $\mathrm{HAH}$. Individuals carrying both mutant type "AG/GG" genotype of rs4953354 and mutant type "CT/CC" genotype of rs6520015 showed a lower risk of HAH.

We detected a HAH incidence of $72.6 \%$ after high-altitude exposure in the current study, slightly lower than the incidence in previous studies $[3,4]$. One possible reason is that subjects are general young soldiers, and they take exercise and military training regularly, resulting in better physical fitness and hypoxia tolerance. Differences in experiment design and ascending altitude have an impact as well. Total population exhibited significantly higher HR, $\mathrm{SBP}, \mathrm{DBP}$, and lower $\mathrm{SpO}_{2}$ before and after high-altitude exposure, and $\mathrm{HAH}$ patients showed significantly lower $\mathrm{SpO}_{2}$ and higher $\mathrm{HR}$ than non-HAH subjects, which was in accordance with previous researches [9]. To ensure sufficient oxygen supply, the activation of the autonomic nervous system and peripheral chemoreceptors may explain the changes of physiological parameters secondary to high-altitude exposure.

The EPAS1 gene locates on the short arm of chromosome 2 between positions 21 and 16 and encodes the endothelial PAS domain protein 1 (EPAS1), which is an oxygen-sensitive alpha submit of HIF-2. Previous studies suggest that rs13419896, rs4953354, and rs6756667 in EPAS1 are associated with high-altitude adaptation in Tibetans [21, 29, 32]. In our study, rs4953354 and rs6756667 are associated with decreased HAH risk in the Han population. The same genetic markers in both Tibetans and Han population confirmed the existence of an adaptive allele. However, unlike Han, Tibetans undergo natural selection for hundreds of generations, and the "G" allele on rs4953354 and "A" allele on rs6756667 have evolved into major alleles in EPAS1 gene for the adaptation in the high-altitude region [27]. Our results also supported that those Han people who carry adaptive alleles may suffer less altitude sickness such as headache at high altitude. Under hypoxia conditions, a family of HIF transcription factors upregulate the expression level of genes involved in cellular and systemic hypoxia adaptation and play crucial roles in vascular responses $[24,36,37]$. Studies propose that EDN1, a vasoconstricting peptide primarily produced in vascular endothelium, could regulate vascular tone and take part in vascular homeostasis and migraine pathophysiology $[38,39]$. What is more, EDN1 is regulated by HIF, for the expression of EDN1 has been observed to be reduced as the degradation of HIF- $1 \alpha$ and HIF-2 $\alpha$ [40]. Although underlying mechanisms are not determined, we deduce that EPAS1 may contribute to $\mathrm{HAH}$ by regulating the expression of EDN1 through the HIF pathway. Moreover, rs4953354 and rs6756667 locate on the introns of EPAS1, in which the greatest genetic differences between Tibetans and the Chinese Han population exist [27]. Thus, we speculate that rs4953354 and rs6756667, close to the binding sites of several transcription factors of EPAS1, may alter the functions of transcription factors binding sites or be in linkage disequilibrium (LD) with other true functional SNPs, thus affecting EPAS1 expression or regulating HIF relative genes in tissues experiencing hypoxia, and eventually assisting body adapting to the hypoxia condition in Chinese Han population. The rs13419896 may not directly participate in pathophysiological progress of oxygen metabolism and is irrelevant to $\mathrm{HAH}$ in our study. Further investigations should focus on finding more variants in or near EPAS1 and explaining exact physiological mechanism associated with HAH.

The PPARA gene locates on chromosome 22 (46150521 bp-46243756 bp) and encodes peroxisome proliferator-activated receptor alpha $(\operatorname{PPAR} \alpha)$. PPAR $\alpha$, a member of nuclear receptor transcription factors family, is highly expressed in the heart, liver, kidney, and skeletal muscle where energy metabolism is quite active, regulating the lipid metabolism and gluconeogenesis, specifically the fatty acid beta-oxidation in mitochondrial and peroxisomal [41]. Hypoxia condition enhances the procedure of anaerobic glycolysis and lactic acid accumulation in venous blood that is regulated by $\operatorname{PPAR} \alpha$ to enhance the utilization ratio of oxygen and keep the brain supplied with sufficient ATPs [42]. Decreased fatty acid oxidation in skeletal muscle is observed in Sherpa highlanders and Tibetans because fatty acid oxidation needs more oxygen when producing the same amount of ATPs [42, 43]. A putatively advantageous PPARA haplotype exhibited a significantly positive relationship with decreased expression or activity of $\operatorname{PPAR} \alpha$ and increased serum free fatty acid in Tibetans, demonstrating that PPARA is associated with hypoxia metabolic adaptation [32]. Likewise, PPARA has an effect on vascular function, transcription of target genes, and decreased level of hemoglobin in Tibetans [44]. The rs6520015 "C" allele in PPARA shows a protective effect on the development of HAH in our study, which is different from the adaptation markers in Tibetans. The SNP locates on the introns of PPARA and is likely to be a noncoding variant. One possible explanation is that rs6520015 affects transcription regulation itself or may be in LD with a truly functional variant in a promoter or an enhancer element of PPARA and alter the expression level of PPARA as an expression quantitative trait locus. Under hypoxia conditions, microRNAs (miRNAs) are observed to be increased significantly in Tibetans than those in the Han population and strongly associated with red blood cell counts, hemoglobin concentration, 
and plasma concentration of erythropoietin [45]. miRNAs, a class of molecules which are approximate 22 nucleotides in length, belong to noncoding RNAs that regulate target gene expression at the posttranscriptional level. Moreover, PPARA is a potential target gene of miR$302 \mathrm{~b}-5 \mathrm{p}$. Combined with the phenomenon that rs7292407 and rs6520015 exhibit a strong LD and the haplotype "CC" decreases HAH risk, although 2 SNPs locate $90 \mathrm{~kb}$ away from the transcription initiation site of PPARA, we extrapolate that the haplotype might change the structure of miRNA and further regulate the expression level of PPARA and its target genes, leading to the development of $\mathrm{HAH}$ at high altitude. According to our results, further investigations should focus on revealing the exact mechanism of how PPARA gene variants regulate the development of HAH.

The present study has several limitations. Firstly, 580 subjects enrolled in our research are Chinese Han young males and they are soldiers on military assignments. Age bias and sex bias may exist, and whether the results could be applied to all populations needs to be verified. Secondly, the diagnosis of $\mathrm{HAH}$ satisfied the criteria of the International Classification of Headache Disorders basically because it is difficult to record the detailed description of headache characteristics in a large field-based study. Subjects suffering $\mathrm{HAH}$ were not transferred to low-altitude immediately after the headache occurred because of military duty, but all $\mathrm{HAH}$ patients recovered after resting for four or five days at $3700 \mathrm{~m}$ with a follow-up observation. Lastly, exact mechanisms of how these SNPs affect genes function and pathophysiological process of $\mathrm{HAH}$ need to be verified by electrophoretic mobility shift assays and dual-luciferase reporter system in future studies.

\section{Conclusion}

We first demonstrated that rs4953354 and rs6756667 in EPAS1 and rs6520015 in PPARA were significantly associated with decreased risk of $\mathrm{HAH}$ in the Chinese Han population. Our research examined the genetic characteristics of $\mathrm{HAH}$ and may evoke further investigation for underlying mechanisms involved in $\mathrm{HAH}$ etiology including functional characterization of transcription and expression. Findings also contributed to screening susceptible populations before ascending to high altitude and offering thinking for prevention and treatment strategies of $\mathrm{HAH}$.

\section{Data Availability}

The data used to support the findings of this study are available from the corresponding author upon request.

\section{Ethical Approval}

The study was reviewed and approved by the Ethics Committee of Xinqiao Hospital of Army Medical University (identification code: 2012014 approved on 9 May 2012). Each participant was informed of the study purpose and whole procedure in detail and signed written informed consent freely and voluntarily before blood collection and further examinations.

\section{Conflicts of Interest}

The authors declare that there are no conflicts of interest.

\section{Authors' Contributions}

Yang Shen and Jihang Zhang contribute equally to this manuscript, and they are co-first author. Lan Huang, Jihang Zhang, and Xubin Gao conceived and designed the experiments. Yang Shen, Jie Yang, Shizhu Bian, and Chen Zhang performed the field experiment and collected data both at sea level and in Lhasa. Chuan Liu and Jie Yu completed the basic experiment. Yang Shen and Jihang Zhang analyzed the data and wrote the paper. Lan Huang critically reviewed and modified the manuscript. All authors approved the final manuscript. All authors appreciated all the participants and their supports in Lhasa.

\section{Acknowledgments}

The authors are grateful to all subjects who took part in this study for their support. The authors thank Mengyang Deng, Can Chen, and Huali Kang for laboratory technical support. They are also grateful for comments from anonymous reviewers which are valuable for improving the manuscript. This study was supported by the National Natural Science Foundation of China (grant nos. 81730054 and 81800396), the Ministry of Health of P.R. China (grant no. 201002012), and Research Project of PLA (grant no. BLJ18J007).

\section{Supplementary Materials}

Supplement Table 1: association between other SNPs and HAH under multiple models. Figure S1: haplotype block map for tag SNPs in EPAS1. (Supplementary Materials)

\section{References}

[1] M. Serrano-Dueñas, "High-altitude headache," Journal of Expert Review of Neurotherapeutics, vol. 7, pp. 245-248, 2007.

[2] K. Zafren, M. Pun, N. Regmi et al., "High altitude illness in pilgrims after rapid ascent to $4380 \mathrm{M}$," Travel Medicine and Infectious Disease, vol. 16, pp. 31-34, 2017.

[3] M. J. Marmura and P. B. Hernandez, "High-altitude headache," Current Pain and Headache Reports, vol. 19, no. 5, p. 483, 2015.

[4] F. J. Carod-Artal, "High-altitude headache and acute mountain sickness," Neurologia, vol. 29, no. 9, pp. 533-540, 2014.

[5] R. C. Roach, P. H. Hackett, O. Oelz et al., "The 2018 lake louise acute mountain sickness score," High Altitude Medicine \& Biology, vol. 19, no. 1, pp. 4-6, 2018.

[6] Headache Classification Subcommittee of the International Headache Society, "The international classification of headache disorders," Cephalalgia, vol. 24, no. Suppl. 1, pp. 9-160, 2004.

[7] J. I. Lopez, A. Holdridge, and J. E. Mendizabal, "Altitude headache," Current Pain and Headache Reports, vol. 17, no. 12, p. 383, 2013. 
[8] E. Silber, P. Sonnenberg, D. J. Collier, A. J. Pollard, D. R. Murdoch, and P. J. Goadsby, "Clinical features of headache at altitude: a prospective study," Neurology, vol. 60, no. 7, pp. 1167-1171, 2003.

[9] M. Burtscher, K. Mairer, M. Wille, and G. Broessner, "Risk factors for high-altitude headache in mountaineers," Cephalalgia, vol. 31, no. 6, pp. 706-711, 2011.

[10] G. Broessner, J. Rohregger, M. Wille, P. Lackner, J.-P. Ndayisaba, and M. Burtscher, "Hypoxia triggers highaltitude headache with migraine features: a prospective trial," Cephalalgia, vol. 36, no. 8, pp. 765-771, 2016.

[11] M. J. Poulin, M. Fatemian, J. G. Tansley, D. F. O’Connor, and P. A. Robbins, "Changes in cerebral blood flow during and after $48 \mathrm{~h}$ of both isocapnic and poikilocapnic hypoxia in humans," Experimental Physiology, vol. 87, no. 5, pp. 633-642, 2002.

[12] S. C. Erzurum, S. Ghosh, A. J. Janocha et al., "Higher blood flow and circulating NO products offset high-altitude hypoxia among Tibetans," Proceedings of the National Academy of Sciences, vol. 104, no. 45, pp. 17593-17598, 2007.

[13] M. B. Vestergaard, U. Lindberg, N. J. Aachmann-Andersen et al., "Acute hypoxia increases the cerebral metabolic rate-a magnetic resonance imaging study," Journal of Cerebral Blood Flow \& Metabolism, vol. 36, no. 6, pp. 1046-1058, 2016.

[14] R. T. Ross, "The random nature of cerebral mountain sickness," The Lancet, vol. 325, no. 8435, pp. 990-991, 1985.

[15] L. J. Norcliffe, M. Rivera-Ch, V. E. Claydon et al., "Cerebrovascular responses to hypoxia and hypocapnia in highaltitude dwellers," The Journal of Physiology, vol. 566, no. 1, pp. 287-294, 2005.

[16] S.-Z. Bian, J.-H. Zhang, X.-B. Gao et al., "Risk factors for highaltitude headache upon acute high-altitude exposure at $3700 \mathrm{~m}$ in young Chinese men: a cohort study," The Journal of Headache and Pain, vol. 14, p. 35, 2013.

[17] S.-Z. Bian, J. Jin, Q.-N. Li et al., "Hemodynamic characteristics of high-altitude headache following acute high altitude exposure at $3700 \mathrm{~m}$ in young Chinese men," The Journal of Headache and Pain, vol. 16, no. 1, p. 527, 2015.

[18] W.-Y. Guo, S.-Z. Bian, J.-H. Zhang et al., "Physiological and psychological factors associated with onset of highaltitude headache in Chinese men upon acute high-altitude exposure at $3700 \mathrm{~m}$," Cephalalgia, vol. 37, no. 4, pp. 336-347, 2017.

[19] E. Garrido, R. Segura, A. Capdevila, J. Pujol, C. Javierre, and J. L. Ventura, "Are himalayan sherpas better protected against brain damage associated with extreme altitude climbs?" Clinical Science, vol. 90, no. 1, pp. 81-85, 1996.

[20] T. Wu and B. Kayser, "High altitude adaptation in Tibetans," High Altitude Medicine \& Biology, vol. 7, no. 3, pp. 193-208, 2006.

[21] T. S. Simonson, Y. Yang, C. D. Huff et al., "Genetic evidence for high-altitude adaptation in Tibet," Science, vol. 329, no. 5987, pp. 72-75, 2010.

[22] L.-D. Ji, Y.-Q. Qiu, J. Xu et al., "Genetic adaptation of the hypoxia-inducible factor pathway to oxygen pressure among eurasian human populations," Molecular Biology and Evolution, vol. 29, no. 11, pp. 3359-3370, 2012.

[23] T. S. Simonson, D. A. McClain, L. B. Jorde, and J. T. Prchal, "Genetic determinants of Tibetan high-altitude adaptation," Human Genetics, vol. 131, no. 4, pp. 527-533, 2012.

[24] A. W. Bigham and F. S. Lee, "Human high-altitude adaptation: forward genetics meets the HIF pathway," Genes \& Development, vol. 28, no. 20, pp. 2189-2204, 2014.
[25] F. R. Lorenzo, C. Huff, M. Myllymäki et al., "A genetic mechanism for Tibetan high-altitude adaptation," Nature Genetics, vol. 46, no. 9, pp. 951-956, 2014.

[26] X. Yi, Y. Liang, E. Huerta-Sanchez et al., "Sequencing of 50 human exomes reveals adaptation to high altitude," Science, vol. 329, no. 5987, pp. 75-78, 2010.

[27] Y. Peng, Z. Yang, H. Zhang et al., "Genetic variations in Tibetan populations and high-altitude adaptation at the Himalayas," Molecular Biology and Evolution, vol. 28, pp. 1075-1081, 2011.

[28] C. M. Beall, G. L. Cavalleri, L. Deng et al., "Natural selection on EPAS1 (HIF2) associated with low hemoglobin concentration in Tibetan highlanders," Proceedings of the National Academy of Sciences, vol. 107, no. 25, pp. 11459-11464, 2010.

[29] M. Hanaoka, Y. Droma, B. Basnyat et al., "Genetic variants in EPAS1 contribute to adaptation to high-altitude hypoxia in Sherpas," PLoS One, vol. 7, no. 12, Article ID e50566, 2012.

[30] Y. Chen, C. Jiang, Y. Luo, F. Liu, and Y. Gao, "An EPAS1 haplotype is associated with high altitude polycythemia in male Han Chinese at the Qinghai-Tibetan plateau," Wilderness \& Environmental Medicine, vol. 25, no. 4, pp. 392-400, 2014.

[31] M. A. Abdelmegeed, K.-H. Moon, J. P. Hardwick, F. J. Gonzalez, and B.-J. Song, "Role of peroxisome proliferator-activated receptor- $\alpha$ in fasting-mediated oxidative stress," Free Radical Biology and Medicine, vol. 47, no. 6, pp. 767-778, 2009.

[32] R.-L. Ge, T. S. Simonson, R. C. Cooksey et al., "Metabolic insight into mechanisms of high-altitude adaptation in Tibetans," Molecular Genetics and Metabolism, vol. 106, no. 2, pp. 244-247, 2012.

[33] E. Zhang, J. Zhang, J. Jin, J. Qin, H. Li, and L. Huang, "Variants of the low oxygen sensors EGLN1 and HIF-1AN associated with acute mountain sickness," International Journal of Molecular Sciences, vol. 15, no. 12, pp. 21777-21787, 2014.

[34] T. Tashi, N. Scott Reading, T. Wuren et al., "Gain-of-function EGLN1 prolyl hydroxylase (PHD2 D4E:C127S) in combination with EPAS1 (HIF-2 $\alpha$ ) polymorphism lowers hemoglobin concentration in Tibetan highlanders," Journal of Molecular Medicine, vol. 95, no. 6, pp. 665-670, 2017.

[35] C.-J. Hu, L.-Y. Wang, L. A. Chodosh, B. Keith, and M. C. Simon, "Differential roles of hypoxia-inducible factor 1 (HIF-1) and HIF-2 in hypoxic gene regulation," Molecular and Cellular Biology, vol. 23, no. 24, pp. 9361-9374, 2003.

[36] G. L. Semenza, "Hypoxia-inducible factors in physiology and medicine," Cell, vol. 148, no. 3, pp. 399-408, 2012.

[37] H. Tian, S. L. McKnight, and D. W. Russell, "Endothelial PAS domain protein 1 (EPAS1), a transcription factor selectively expressed in endothelial cells," Genes \& Development, vol. 11, no. 1, pp. 72-82, 1997.

[38] J. Miao, F. Wang, and Y. Fang, "Association of 231G $>$ A polymorphism of endothelin type A receptor gene with migraine: a meta-analysis," Journal of the Neurological Sciences, vol. 323, no. 1-2, pp. 232-235, 2012.

[39] R. M. Gupta, J. Hadaya, A. Trehan et al., "A genetic variant associated with five vascular diseases is a distal regulator of endothelin-1 gene expression," Cell, vol. 170, no. 3, pp. 522-533, 2017.

[40] Y. Pang, C. Yang, J. Schovanek et al., "Anthracyclines suppress pheochromocytoma cell characteristics, including metastasis, through inhibition of the hypoxia signaling pathway," Oncotarget, vol. 8, no. 14, pp. 22313-22324, 2017. 
[41] S. W. Standage, B. G. Bennion, T. O. Knowles et al., "PPAR $\alpha$ augments heart function and cardiac fatty acid oxidation in early experimental polymicrobial sepsis," American Journal of Physiology-Heart and Circulatory Physiology, vol. 312, no. 2, pp. H239-H249, 2017.

[42] F. Kinota, Y. Droma, N. Kobayashi et al., "The contribution of genetic variants of the peroxisome proliferator-activated receptor-alpha gene to high-altitude hypoxia adaptation in Sherpa highlanders," High Altitude Medicine \& Biology, Epub ahead of print, 2018.

[43] R.-L. Ge, T. S. Simonson, V. Gordeuk, J. T. Prchal, and D. A. McClain, "Metabolic aspects of high-altitude adaptation in Tibetans," Experimental Physiology, vol. 100, no. 11, pp. 1247-1255, 2015.

[44] V. Anttila, B. S. Winsvold, P. Gormley et al., "Genome-wide meta-analysis identifies new susceptibility loci for migraine," Nature Genetics, vol. 45, no. 45, pp. 912-917, 2013.

[45] Y. Yan, Y. Shi, C. Wang et al., "Influence of a high-altitude hypoxic environment on human plasma microRNA profiles," Scientific Reports, vol. 5, no. 1, p. 15156, 2015. 